IgA1 antibodies specific for outer membrane protein PorA modulate the interaction between Neisseria meningitidis and the epithelium

R.E. Horton ${ }^{1,3^{*}}$, G. Vidarsson ${ }^{2,4}$, M. Virji ${ }^{1}$, N.A. Williams ${ }^{1}$, and R.S. Heyderman ${ }^{1,5}$

${ }^{1}$ Department of Cellular and Molecular Medicine, School of Medical Sciences, University of Bristol, Bristol, UK

${ }^{2}$ Department of Immunology, University Hospital Utrecht, Utrecht, The Netherlands

Running Title: Effect of IgA on meningococcal-epithelial interactions

Word count: Abstract -150

Text $-3,678$

*Corresponding author / reprint requests: Rachel Horton, Institute for Glycomics, Griffith University Gold Coast, QLD 4222, Australia

Telephone: +61 755529351

Email: r.horton@griffith.edu.au 


\section{Current affiliations:}

${ }^{3}$ Institute of Glcomics, Griffith University Gold Coast, QLD 4222, Australia

${ }^{4}$ Department of Experimental Immunohaematology, Sanquin Research and Landsteiner Laboratory, Academic Medical Center, University of Amsterdam, Plesmanlaan 125, 1066 CX Amsterdam, The Netherlands

${ }^{5}$ Malawi-Liverpool-Wellcome Trust Clinical Research Programme, PO Box 30096, Chichiri, Blantyre 3, Malawi 


\section{ABSTRACT}

Despite high carriage rates of Neisseria meningitidis, incidence of meningococcal disease remains low, partially due to development of natural immunity. We have previously demonstrated an inverse relationship between salivary anti-meningococcal IgA and disease incidence, but little is known about the contribution of IgA to immunity at mucosal surfaces. Here we show strong immunoreactivity by human salivary IgA against the meningococcal outer membrane porin, PorA. Monomeric anti-PorA IgA1 (humanized chimeric antibodies) but not IgG increased the association of unencapsulated serogroup B N. meningitidis (H44/76) with Chang (conjunctival) but not with either Detroit (pharyngeal) cells or with A549 (alveolar) epithelial cells. Association of encapsulated N. meningitidis was not increased. Epithelial binding of IgA was Fc fragment dependent and not inhibited by IgM. Together these data suggest the presence of a specific epithelial IgA receptor that could influence the effect of both naturally acquired and vaccine induced IgA antibodies at the epithelial surface.

Key words: Immunoglobulin A, Neisseria meningitidis, mucosal immunity, antibodies, PorA 


\section{INTRODUCTION}

Neisseria meningitidis is an important cause of meningitis in children and young adults worldwide [1,2]. However, although asymptomatic carriage is frequent [3], the development of meningococcal disease is relatively rare. In the UK it has been estimated that most individuals experience up to 10 meningococcal carriage episodes during their lifetime [4], while disease develops in only 2-5 individuals per 100,000 per year. The disparity between frequency of carriage and disease may be partly accounted for by the low virulence of many carriage strains but also suggests a role for naturally acquired protective immunity $[2,5,6]$.

Naturally developed immunity is believed to occur through multiple colonization events at the mucosal surface by $N$. meningitidis, related organisms such as Neisseria lactamica and other antigenically cross reacting flora [7]. There is considerable evidence that either naturally acquired or vaccine-induced circulating complement fixing IgG antibody, high in bactericidal activity, protects against invasive disease $[1,6,8,9]$. We have previously demonstrated T-cell mediated immunological memory to $N$. meningitidis at the mucosal level which has a predominately proinflammatory phenotype. We have shown that the magnitude of these responses is highly regulated and increases with age $[10,11]$. IgA is the major antibody at mucosal surfaces in the upper respiratory tract and mucosal clearance through the elaboration of secretory IgA is considered to be important in limiting meningococcal colonization and preventing early invasion. We have previously reported a link between salivary antimeningococcal IgA levels and the incidence of meningococcal infection in the population [2]. However, the target meningococcal antigens for this IgA and the mechanisms of this immunity are uncertain.

IgA consists of two subclasses, IgA1 and IgA2, differing almost exclusively by a 13 amino acid sequence present in the hinge region of IgA1 [12], the most common isotype at most mucosal surfaces [13]. The hinge region is the site of cleavage for IgA1 proteases expressed 
by a number of pathogens including $N$. meningitidis and Streptococcus pneumonia $[14,15]$. Serum IgA is mainly monomeric whereas mucosal IgA is mostly dimeric, synthesized by B cells located in the mucosal associated lymphoid tissue (MALT) [16]. During transcytosis of IgA through epithelial cells, the extracellular domain of the receptor for dimeric IgA, the polymeric Ig receptor (PIgR), is cleaved from the cells (secretory component) and remains complexed with IgA (secretory IgA).

We created a panel of humanized chimeric antibodies with identical mouse V regions, and different human constant (C) regions against a single PorA epitope (P1.16 in surface loop four) [17]. Meningococcal PorA is a membrane spanning protein with eight well conserved surface exposed loops [18] with the exception of two hyper variable regions (VR1 and VR2) [19]. While chimeric IgG1 and IgG3 were both good activators of complement mediated lysis, neither IgA1 nor IgA2 activated complement but blocked IgG mediated killing in a concentration dependent manner. In the present study we have shown strong immunoreactivity by human salivary IgA against the meningococcal outer membrane porin, PorA. We demonstrate that anti-PorA IgA1 antibodies increase meningococcal association with Chang epithelial cells and suggest that this interaction occurs through a specific IgA receptor.

\section{RESULTS}

\subsection{Anti-PorA IgA is highly represented in human saliva}

We have previously reported the presence of IgA to a range of meningococcal outer membrane proteins saliva samples obtained from adults and children [2]. Subsequent experiments were carried out to determine the targets of IgA antibodies. Densitometry analysis of OMV Western blots (Fig 1a), with proteins identified by band size, demonstrated strong immunoreactivity of salivary antibodies against PorA and to a lesser extent PorB (Fig 
1b). This was despite very similar levels of PorA and PorB in OMVs [20]. Antibodies to RmpM (putative peptidoglycan binding protein [21]), Opa, Opc and putative OMP P1 were also detected in some subjects (Fig 1a and data not shown).

\subsection{Monomeric IgA1 (mIgA1) but not IgG1 increases meningococcal association with}

\section{Chang epithelial cells}

To determine the potential functional effects of anti-PorA antibody on meningococcal interactions with the mucosal barrier, we initially co-cultured with H44/76 (P1.7,16-2 serosubtype) with humanised mIgA1 antibody and Chang epithelial cells. The chimeric antibody is highly specific for the PorA surface loop four expressed by this strain and does not bind another commonly used serogroup B clinical strain N. meningitidis MC58 (B.15.P1.7,16 immunotype L3) with a single D to $\mathrm{N}$ amino acid substitution in the DTNNN consensus sequence (results not shown) [22]. The Chang cell line was selected because it expresses low levels of CEACAMs which are potent mediators of Opa-dependent epithelial adherence and invasion [23-25]. Time course experiments using an unencapsulated H44/76 derivative revealed an increase in meningococcal-epithelial association over three hours which was further enhanced by specific mIgA1 (Fig 2a) $\left(9.881 \times 10^{4}\right.$ cfu / well \pm sem 1.9 x $10^{4}$ vs $16.97 \times 10^{4} \mathrm{cfu} /$ well \pm sem $0.7813 \times 10^{4}$ with specific mIgA1, $\left.\mathrm{P}=0.0435\right)$. This antibody-mediated increase in association was not seen with humanised IgG specific for the same epitope of the PorA loop region (Fig 2b) (sem=0.5025 \pm 4.334$)$. Association assays using $\mathrm{F}(\mathrm{ab})_{2}$ fragments showed that the effects of mIgA1 on meningococcal association with Chang cells are Fc fragment dependent (Fig 2b).

It has previously been shown that capsulation reduces adherence of meningococci to Chang epithelial cells [24-26]. In this study the effect of monomeric IgA1 (mIgA1) on the capsulated 
parent $N$. meningitidis H44/76 strain was examined but no increase in epithelial association was observed.

\subsection{Modulation of meningococcal association with other epithelial cells by mIgA1}

To investigate the influence of anti-PorA mIgA on meningococcal interactions with epithelial cells expressing intermediate and high levels of CEACAM, association experiments were conducted with A549 (alveolar) and Detroit (pharyngeal carcinoma) cells characterised for their receptor expression previously. Mean fluorescence intensity for CEACAM expression was approximately 5 for A549 cells, compared with 1 for Chang cells and 22 for Detroit cells [25]. Although pre-incubation of unencapsulated H44/76 with specific mIgA1 resulted in a two-fold increase in association with Chang cells $\left(29.29 \times 10^{4} \mathrm{cfu} /\right.$ well vs 66.35 x $10^{4} \mathrm{cfu} /$ well with specific mIgA1, $\mathrm{P}=0.05)$, significant increases with A549 cells (82.16 x $10^{4}$ vs 87.4 x $10^{4}$ respectively, $\left.\mathrm{P}=0.7535\right)$ or Detroit cells $\left(91.42 \times 10^{4}\right.$ vs $89.3 \times 10^{4}$, respectively, $\mathrm{P}=0.9314$ ) were not seen (Fig 3).

\subsection{Characteristics of IgA binding to Chang cells}

Further analysis of IgA binding to Chang was carried out using commercially available serum derived IgA (mainly monomeric, IgA1 and IgA2), colostrum derived IgA (mainly polymeric, IgA1 and IgA2). Both serum and colostrum derived IgA bound to a sub-population (approximately 22\%) of Chang cells (Fig 4a\&b). Chimeric anti-PorA mIgA1 was also shown to bind Chang cells similarly whereas human serum-derived IgG did not (data not shown). To investigate the possibility that meningococci upregulate IgA receptor expression on epithelial cells, Chang cells were pre-incubated in the presence of unencapsulated H44/76 meningococci for three hours. No change in serum derived IgA binding was seen (Fig 4c\&d). In order to determine whether the state of the cell cycle influences binding of IgA to Chang 
cells, thereby providing a possible explanation for the observation that IgA only binds to 20$45 \%$ of the total Chang cell population, previous experiments were repeated following treatment with the cell cycle inhibitor, aphidicolin [27]. This treatment approximately doubled the amount of serum derived IgA1 binding to Chang cells (Fig 4e\&f).

\subsection{Role of known IgA receptors in IgA binding to Chang cells}

Several IgA receptors have been described on epithelial cells which bind mIgA, pIgA or both [28]. To assess which receptor might be involved, binding competition experiments were conducted. No interference between serum and colostrum derived IgA were seen, even at high concentrations of competing IgA (Fig 5a). In order to investigate the possibility that pIgA may bind to Chang cells via the J chain, competition experiments were conducted with IgM which is a pentameric immunoglobulin, also possessing a $\mathrm{J}$ chain. These demonstrated that IgM was unable to inhibit binding of colostrum derived IgA to Chang cells (Fig 5b).

The asialoproteoglycan receptor (ASPGR) binds to terminal galactose and N-acetyl-Dgalactosamine (NAG) residues found on IgA [29]. It is therefore possible to inhibit binding of IgA by adding its natural ligand, NAG. However in this study, NAG did not inhibit IgA binding to Chang cells, even at concentrations of $1 \mathrm{mg} / \mathrm{ml}$ (Fig 5c). To explore the possibility that the FcaR or the polymeric IgA receptor (pIgR) mediate the IgA binding observed RTPCR was carried out. mRNA for both receptors was shown to be absent in Chang cells (Fig 6), which implies that neither receptor is expressed.

\section{DISCUSSION}

IgA is present in abundant quantities in mucosal secretions [30] and is widely believed to prevent colonisation and invasion by upper respiratory tract bacteria such as $N$. meningitidis. 
However, little is known regarding its mechanisms of action. IgA activates the classical complement pathway poorly $[17,31]$ and has traditionally been viewed as anti-inflammatory, blocking the mucosal effects of microbial antigens through three major mechanisms. Firstly, the prevention of attachment and invasion by micro-organisms by surrounding them with a hydrophilic shell, interference with motility due to interactions with flagella [32], or neutralising bacterial products [33]. Secondly, binding antigens and transporting them through the epithelium, thus eliminating excess antigens from the mucosa. Thirdly, interception of antigens during viral transcytosis and intracellular neutralisation by interference with viral assembly or synthesis [34]. In relation to the meningococcus, research in this area has been hampered by the lack of good laboratory models and the paucity of related reagents. In this study, we have used chimeric antibodies specific for $N$. meningitidis PorA to investigate their role at the mucosal epithelium. We show that in Chang epithelial cells which express low levels of CEACAMs (an important receptor for Opa-mediated meningococcal adherence and invasion) [25], anti-PorA IgA but not IgG leads to an increase in meningococcal association but not invasion. In contrast, with A549 (alveolar) and Detroit (pharyngeal) epithelial cells which express higher levels of CEACAMs, anti-PorA IgA does not increase meningococcalepithelial cell association. PorA is not known to mediate direct interactions with host cells but the finding of mucosal IgA specific for PorA together with evidence that anti-PorA IgA enhances meningococcal interaction with some epithelia implicates this IgA in either immune clearance or a novel colonisation mechanism.

$N$. meningitidis shows considerable tropism for upper respiratory tract epithelium $[35,36]$. Many carried strains are unencapsulated [37] and our data suggests that compared to encapsulated meningococci, these are most susceptible to anti-PorA IgA1-enhanced epithelial cell association, presumably through greater exposure of the PorA antigen. However, we found no evidence that meningococci upregulated IgA binding either directly or indirectly 
(unpublished observation) as has been seen with other receptor pathways. The inter-cell line differences described here may reflect the highly variable expression of different IgA surface receptors or differential usage of the same receptor. However, we suggest that CEACAM-Opa interactions which are responsible for significantly greater adhesion to A549 compared with Chang cell lines used in current studies [23] may have masked the influence of anti-PorA mIgA. Opa proteins are subject to phase variation [38] and CEACAMs may vary in receptor density [25]. Follow-on studies are therefore investigating whether primary epithelial cells from anatomically relevant locations display similar IgA binding and mIgA-meningococcal interactions. Further antigen-specific IgA reagents are required for the investigation of mucosal immunity to other key meningococcal antigens.

The anti-PorA IgA mediated increases in meningococcal adhesion to Chang cells appear to occur via a specific IgA receptor. It is unlikely that this represents a non-specific interaction as PorA-specific IgG did not enhance binding, increased binding was Fc fragment dependent and IgA binding was not inhibited by IgM. Several known IgA receptors including the Fc $\alpha / \mu \mathrm{R}$ [39], pIgR, Fc $\alpha \mathrm{R}$ and ASPGR have been excluded. The transferrin receptor (Tfr) [40] and several largely uncharacterised receptors [41-44] have also been shown to bind IgA. We determined that Chang cells express $\mathrm{Tfr}$ (data not shown) but the role of $\mathrm{Tfr}$ and the possibility of additional novel receptor(s) in this setting remain to be confirmed. The plasma membrane recycling system regulates the return to the plasma membrane of internalized receptors, transporters and channels. As cells undergo cell division, nonessential pathways, including plasma membrane recycling, are disabled. Characterisation of the functional phenotype of the IgA receptor implicated in our studies revealed IgA binding to be influenced by cell cycle stage. It is unlikely that aphidicolin treatment resulted in cells maintained in a $\mathrm{G}_{0}$ type resting state with increased receptor expression, as following treatment, the IgA-positive 
population of epithelial cells was not able to bind more IgA. It is more likely that plasma membrane and therefore receptor recycling was maximized in these epithelial monolayers as they approached confluence. Whatever the mechanism, it is clear that surface expression is affected by the state of the cell cycle.

The receptor(s)-mediated adherence of IgA-bound meningococci to epithelial cells could have several outcomes depending on which surface (apical/basolateral) the receptor is expressed on, whether bacteria are internalised, and, if internalised, they are transported back through the cell onto the cell surface. If the receptor remains on the cell surface, this may be a method of holding potentially pathogenic meningococci at the epithelium in order to prevent their proliferation and subsequent invasion. This would support our previously reported data demonstrating an inverse correlation between anti-meningococcal IgA and disease incidence [2], suggesting a protective role for IgA at the mucosal surface. However, a number of studies have shown that mucosal IgA rather than being protective actually serves to increase microbial colonisation and invasion. Epstein-Barr virus (EBV) infection of epithelial cells, for example, is dependent upon transport in complexes with anti-EBV IgA by the pIgR [45]. IgA against the capsule of $S$. pneumoniae increases association of the bacteria with epithelial cells [46]. This increase in pneumococcal association is only seen when the Fc portion is cleaved off by the IgA1 protease, contrasting with what we now report with $N$. meningitidis. The chimeric IgA1 used in these experiments is susceptible to IgA protease and it has previously been shown that its cleavage leads to the release of a proportion of bound Fab fragments [47] which would explain why we were unable to demonstrate an attenuation of meningococcal association when using a strain in which the IgA protease has been knocked out (unpublished observation). 


\subsection{Conclusions}

In conclusion, naturally-acquired anti-PorA IgA is prominent in human saliva. Our data have shown that anti-PorA IgA increases meningococcal-epithelial adhesion with Chang cells and suggest that anti-meningococcal IgA may regulate commensal colonisation and prevent invasion via specific IgA epithelial receptor(s). However, our findings do not preclude the possibility that meningococci may subvert the immune system to use IgA and its receptors to aid colonisation and invasion. The next step is clearly the use of primary nasopharyngeal epithelial cells in association assays in order to elucidate the biological significance of the data presented here, and the precise mechanism in an ex-vivo scenario. We have previously shown that systemic vaccination with MenB vaccines does not increase meningococcalspecific salivary IgA [48]. Nonetheless, the precise role of mucosal IgA against meningococcal surface antigens needs to be determined in order to inform future vaccine strategies against $N$. meningitidis and other mucosal pathogens.

\section{MATERIALS AND METHODS}

\subsection{Bacterial Strains and Growth Conditions}

Serogroup B meningococcal strains used in this investigation were MC58 (B15:P1.7,16;L3,7,9) [49], TR4 (B15:P7-2,4;L3,7,9), H44/76 (B15:P1.7,16;L3,7,9) [50] and a spontaneous unencapsulated H44/76 mutant. All strains were grown from frozen glycerol stocks and used after overnight growth on BHI agar supplemented with $10 \%$ horse blood at $37^{\circ} \mathrm{C}, 5 \% \mathrm{CO}_{2}$.

\subsection{Cell Lines and Culture Conditions}

Human epithelial cell lines were grown in the following media: Chang conjunctival epithelial cells, Medium 199; A549 lung pneumocyte cells, F-12 HAM nutrient medium and Detroit 
562 pharyngeal cells, minimum essential Eagle’s medium, 1 mM sodium pyruvate, 1\% nonessential amino acids. All media were supplemented with 10\% FCS, 4mM L-Glutamine and $100 \mu \mathrm{g} / \mathrm{ml}$ penicillin/streptomycin and grown at $37^{\circ} \mathrm{C}, 5 \% \mathrm{CO}_{2}$. Media components were from Invitrogen (CA, USA) unless otherwise stated.

\subsection{SDS-PAGE of outer membrane vesicles (OMVs) and Western Blotting}

For SDS-PAGE and Western blotting [51], OMVs (kind gifts from RIVM (The Netherlands)) [10] in NuPAGE LDS sample buffer, were boiled briefly and loaded onto a NuPAGE 10\% Bis-Tris pre-cast gel (Invitrogen), and run at $180 \mathrm{~V}$ for 1 hour. Transfer was carried out after soaking in transfer buffer (2.91g Trizma base, $1.47 \mathrm{~g}$ glycine, $0.188 \mathrm{~g}$ SDS, $100 \mathrm{ml}$ methanol in 400mls distilled water) using a Trans-blot semi-dry transfer cell (Bio-Rad) for 15 minutes, 15V. The gel was stained using Gel Code blue stain reagent (Pierce, Rockford, USA). Nitrocellulose was blocked overnight at $4^{\circ} \mathrm{C}$ with PBS, 5\% BSA. Saliva samples collected from healthy adults and children in Bristol (as a source of antibodies) were incubated with the blot for 2 hours, RT. After washing, alkaline phosphatase conjugated goat anti-human IgA was added and incubated for 1 hour, RT. The blot was developed with Nitro blue tetrazolium chloride/5-Bromo-4-Chloro-3-indolyl phosphate (NBT/BCIP), and the reaction stopped with water. Band densities were measured using Scion Image software (Scion Corporation, Frederick, USA).

\subsection{Chimeric Antibodies}

BHK cell lines producing chimeric IgG and IgA-anti PorA P1.16 [17] were grown in RPMI1640 supplemented with $10 \%$ FCS, 200mM L-Glutamine, $100 \mu \mathrm{g} / \mathrm{ml}$ Penicillin/Streptomycin, $100 \mu \mathrm{M}$ methotrexate, and $75 \mu \mathrm{M} \mathrm{ZnCl}_{2}$. IgA antibodies with identical mouse variable regions and different human constant regions, directed against the 
single PorA epitope P1.16 were purified using immobilized jacalin (Pierce, Rockford, IL, USA) on an XK 16 column (Amersham Biosciences). Fractions were desalted on a PD-10 column (Amersham), and separated on a superdex 200 column run in conjunction with the AKTA system (Amersham). Concentration of IgA was determined by ELISA using commercially available human IgA (Sigma) as a standard and predicted molecular weight confirmed by SDS page. Polymeric IgA1 did not assemble appropriately under these conditions and therefore was not used in the experiments detailed below.

\subsection{Generation of $\mathrm{F}(\mathrm{ab})_{2}$ fragments}

Purified chimeric IgA1 was subjected to digestion by pepsin (50mg/ml pepsin (Sigma) in 20mM sodium acetate, $\mathrm{pH} 5.0$ ) to yield $\mathrm{F}(\mathrm{ab})_{2}$ fragments. IgA1 was digested for 60 minutes, RT. The reaction was stopped by addition of $1.5 \mathrm{M}$ Tris- $\mathrm{HCl}, \mathrm{pH} 8.8$ to create a neutral $\mathrm{pH}$. $\mathrm{F}(\mathrm{ab})_{2}$ fragments were separated from the resulting mixture using a jacalin column as described above.

\subsection{Cell Association Assays}

Bacterial association and invasion was measured by viable count assays as previously described [36]. Briefly, meningococci were pre-incubated for ten minutes, RT, with the relevant antibody then incubated with epithelial cell monolayers (approximately 500 meningococci per cell) for three hours, $37^{\circ} \mathrm{C}$. Monolayers were washed and permeabilised using $1 \%$ saponin and released cell-associated bacteria plated onto brain-heart infusion agar supplemented with 5\% heated horse serum (HBHI) for overnight incubation. Colonies were counted and used to estimate the number of cell-associated bacteria from the original inoculum. 
Cell free control wells present in each assay ensure that differences in colony numbers are due to cell binding not association with plastic.

\subsection{RNA extraction and RT-PCR for IgA receptors}

RNA was extracted from confluent cell monolayers using RNAqueous-4PCR (Ambion, Texas, USA), as per manufacturer's instructions. Approximate RNA concentrations were calculated by absorbance at $260 \mathrm{~nm}$ and $280 \mathrm{~nm}$. RT-PCR was performed using the Access RT-PCR system (Promega). First strand cDNA was synthesized using AMV reverse transcriptase and oligo (dT) at $48^{\circ} \mathrm{C}$ for 45 minutes and $42^{\circ} \mathrm{C}$ for 60 minutes for pIgR and FcaR respectively. cDNA was amplified using primers: $p I g R$ forward (5'GGTCCCGAGGAGGTGAATAGTG-3'

$\begin{array}{lllll}\text { GGTCCCGAGGAGGTGAATAGTG-3') } & \text { and } & \text { reverse } & \text { (3’- } \\ \text { CTGACCTCCAGGCTGACATCAA-5') } & \text { [52]; } & F c \alpha R & \text { forward } & \text { (5'- } \\ \text { AAGCTTACCTGACCCAGCTGATG } & - \text { 3’) }^{\prime} & \text { and } & \text { reverse }\end{array}$
AAGCTTCTTAGTGAGCTTTTCTCTC-5') [42]. PCR was carried out in a Peltier thermal cycler (MJ Research, MA, USA). The program for pIgR was: 30 s at $95^{\circ} \mathrm{C}, 60$ s at $55^{\circ} \mathrm{C}$ and $120 \mathrm{~s}$ at $68^{\circ} \mathrm{C}$, total of 50 cycles. The program for $\mathrm{Fc} \alpha \mathrm{R}$ was: $20 \mathrm{~s}$ at $94^{\circ} \mathrm{C}, 30 \mathrm{~s}$ at $56^{\circ} \mathrm{C}$ and 180s at $72^{\circ} \mathrm{C}$, total of 45 cycles. Quantum RNA Universal 18S RNA primers (Ambion) giving rise to a 315bp product were run in parallel with each reaction. PCR products were mixed with a loading dye (Invitrogen) and run on an agarose gel (0.8mg agarose, 100ml TBE buffer, $15 \mu \mathrm{l}$ ethidium bromide) for 45 minutes, 180V. Agarose gels were visualized and photographed under UV light. PCR products requiring sequencing for confirmation were sent to The Sequencing Service, University of Dundee, Scotland. Probable sequence products were then identified using Genomes Fasta (http://www.ebi.ac.uk/fasta33/genomes.html). 


\subsection{Antibody Biotinylation}

Human serum-derived and colostrum-derived IgA (Sigma) were biotinylated for use in flow cytometry using an EZ-Link Sulfo-NHS-Biotinylation kit (Pierce) as per manufacturer's instructions. The level of biotin incorporation was measured by a HABA assay and levels of 11.81 and 3.86 moles biotin per mole IgA were achieved for IgA from serum and colostrum respectively.

\subsection{Flow Cytometry}

Chang cell monolayers were grown to confluency overnight in 24 well plates (Invitrogen) and monolayers were disrupted with Pucks saline and re-suspended in PBS, 10\% FCS [53]. Samples were incubated with biotinylated IgA, with or without a potentially competing antibody for 20 minutes, then washed prior to a further 20 minute incubation with a streptavidin/FITC conjugate (Dako). Cells were fixed with paraformaldehyde and analysed on the FACSCalibur flow cytometer (BD Biosciences) with Win-MDI 2.8 software (The Scripps Research Institute, La Jolla, CA, USA).

\subsection{Cell Cycle Synchronisation}

Aphidicolin, a reversible DNA polymerase inhibitor, was used to block cells at the G1 to S phase transition of the cell cycle, employing a method described by Kues et al. [54]. Chang cells were grown in 24 well plates (Invitrogen) until 60-80\% confluency (logarithmic growth phase) was reached. Growth medium was then replaced with medium containing only $0.2 \%$ FCS, and after a further 48 hours replaced with medium containing 10\% FCS and $6 \mu \mathrm{M}$ Aphidicolin. Cells were then cultured for 14 hours prior to flow cytometry. 


\subsection{Statistical Analysis}

All data analyses were carried out using Prism version 3.0 (GraphPad Software). For association data, the bar charts represent the mean of three wells while the vertical bars show the standard error of the mean (SEM). Different conditions were compared with the control wells using the paired t-test.

\section{ACKNOWLEDGEMENTS}

This study was supported by a research studentship from the Health Protection Agency (Rachel Horton). We are also grateful for the support of Meningitis UK and the Meningitis Research Foundation. Some of the studies were carried out in the Spencer Dayman Meningitis Laboratories.

\section{REFERENCES}

[1] Goldschneider, I., Gotschlich, E.C. \& Artenstein, M.S. Human immunity to the meningococcus. I. The role of humoral antibodies. J Exp Med 1969, 129(6), 13071326.

[2] Horton, R.E., Stuart, J., Christensen, H. et al. Influence of age and carriage status on salivary IgA to Neisseria meningitidis. Epidemiol Infect 2005, 133(5), 883-889.

[3] Caugant, D.A., Hoiby, E.A., Magnus, P. et al. Asymptomatic carriage of Neisseria meningitidis in a randomly sampled population. Journal of Clinical Microbiology 1994, 32(2), 323-330.

[4] Trotter, C.L., Gay, N.J. \& Edmunds, W.J. The natural history of meningococcal carriage and disease. Epidemiol Infect 2006, 134(3), 556-566.

[5] Heyderman, R.S., Davenport, V. \& Williams, N.A. Mucosal immunity and optimizing protection with meningococcal serogroup B vaccines. Trends Microbiol 2006, 14(3), 120-124.

[6] Goldschneider, I., Gotschlich, E.C. \& Artenstein, M.S. Human immunity to the meningococcus. II. Development of natural immunity. J Exp Med 1969, 129(6), 13271348.

[7] Hoff, G.E. \& Hoiby, N. Cross-reactions between Neisseria meningitidis and twentyseven other bacterial species. Acta Pathol Microbiol Scand [B] 1978, 86(2), 87-92.

[8] Balmer, P. \& Borrow, R. Serologic correlates of protection for evaluating the response to meningococcal vaccines. Expert Rev Vaccines 2004, 3(1), 77-87. 
[9] Borrow, R., Carlone, G.M., Rosenstein, N. et al. Neisseria meningitidis group B correlates of protection and assay standardization--international meeting report Emory University, Atlanta, Georgia, United States, 16-17 March 2005. Vaccine 2006, 24(24), 5093-5107.

[10] Davenport, V., Guthrie, T., Findlow, J., Borrow, R., Williams, N.A. \& Heyderman, R.S. Evidence for naturally acquired T cell-mediated mucosal immunity to Neisseria meningitidis. J Immunol 2003, 171(8), 4263-4270.

[11] Davenport, V., Groves, E., Hobbs, C.G., Williams, N.A. \& Heyderman, R.S. Regulation of Th-1 T cell-dominated immunity to Neisseria meningitidis within the human mucosa. Cell Microbiol 2007, 9(4), 1050-1061.

[12] van Egmond, M., Damen, C.A., van Spriel, A.B., Vidarsson, G., van Garderen, E. \& van de Winkel, J.G. IgA and the IgA Fc receptor. Trends Immunol 2001, 22(4), 205211.

[13] Kett, K., Brandtzaeg, P., Radl, J. \& Haaijman, J.J. Different subclass distribution of IgA-producing cells in human lymphoid organs and various secretory tissues. $J$ Immunol 1986, 136(10), 3631-3635.

[14] Mulks, M.H. \& Plaut, A.G. IgA protease production as a characteristic distinguishing pathogenic from harmless neisseriaceae. $N$ Engl J Med 1978, 299(18), 973-976.

[15] Kilian, M., Mestecky, J. \& Schrohenloher, R.E. Pathogenic species of the genus Haemophilus and Streptococcus pneumoniae produce immunoglobulin A1 protease. Infect Immun 1979, 26(1), 143-149.

[16] Mestecky, J. \& McGhee, J.R. Immunoglobulin A (IgA): molecular and cellular interactions involved in IgA biosynthesis and immune response. Adv Immunol 1987, 40, 153-245.

[17] Vidarsson, G., van Der Pol, W.L., van Den Elsen, J.M. et al. Activity of human IgG and IgA subclasses in immune defense against Neisseria meningitidis serogroup B. $J$ Immunol 2001, 166(10), 6250-6256.

[18] van der Ley, P., Heckels, J.E., Virji, M., Hoogerhout, P. \& Poolman, J.T. Topology of outer membrane porins in pathogenic Neisseria spp. Infect Immun 1991, 59(9), 29632971.

[19] McGuinness, B., Barlow, A.K., Clarke, I.N. et al. Deduced amino acid sequences of class 1 protein (PorA) from three strains of Neisseria meningitidis. Synthetic peptides define the epitopes responsible for serosubtype specificity. J Exp Med 1990, 171(6), 1871-1882.

[20] Willams, J.N., Skipp, P.J., Humphries, H.E., Christodoulides, M., O'Connor, C.D. \& Heckels, J.E. Proteomic analysis of outer membranes and vesicles from wild-type serogroup B Neisseria meningitidis and lipopolysaccharide-deficient mutant. Infection and Immunity 2007, 75(3), 1364-1372.

[21] Grizot, S. \& Buchanan, S.K. Structure of the OmpA-like domain of RmpM from Neisseria meningitidis. Mol Microbiol 2004, 51(4), 1027-1037.

[22] van den Elsen, J.M., van Unen, L.M., van Bloois, L. et al. Thermodynamic analysis of the interaction between a bactericidal antibody and a PorA epitope of Neisseria meningitidis. Biochemistry 1997, 36(41), 12583-12591.

[23] Griffiths, N.J., Bradley, C.J., Heyderman, R.S. \& Virji, M. IFN-gamma amplifies NFkappaB-dependent Neisseria meningitidis invasion of epithelial cells via specific upregulation of CEA-related cell adhesion molecule 1. Cell Microbiol 2007, 9(12), 2968-2983.

[24] Virji, M., Evans, D., Hadfield, A., Grunert, F., Teixeira, A.M. \& Watt, S.M. Critical determinants of host receptor targeting by Neisseria meningitidis and Neisseria 
gonorrhoeae: identification of Opa adhesiotopes on the N-domain of CD66 molecules. Mol Microbiol 1999, 34(3), 538-551.

[25] Bradley, C.J., Griffiths, N.J., Rowe, H.A., Heyderman, R.S. \& Virji, M. Critical determinants of the interactions of capsule-expressing Neisseria meningitidis with host cells: the role of receptor density in increased cellular targeting via the outer membrane Opa proteins. Cell Microbiol 2005, 7(10), 1490-1503.

[26] McNeil, G., Virji, M. \& Moxon, E.R. Interactions of Neisseria meningitidis with human monocytes. Microb Pathog 1994, 16(2), 153-163.

[27] Fox, M.H., Read, R.A. \& Bedford, J.S. Comparison of synchronized Chinese hamster ovary cells obtained by mitotic shake-off, hydroxyurea, aphidicolin, or methotrexate. Cytometry 1987, 8(3), 315-320.

[28] Monteiro, R.C. \& Van De Winkel, J.G. IgA Fc receptors. Annu Rev Immunol 2003, 21, 177-204.

[29] Sancho, J., Gonzalez, E. \& Egido, J. The importance of the Fc receptors for IgA in the recognition of IgA by mouse liver cells: its comparison with carbohydrate and secretory component receptors. Immunology 1986, 57(1), 37-42.

[30] Kerr, M. The strucrue and function of human IgA. Biochemical Journal 1991, 271, 285-296.

[31] Kerr, M.A. The structure and function of human IgA. Biochem $J$ 1990, 271(2), 285296.

[32] Heremans, J.F. The IgA system in connection with local and systemic immunity. Adv Exp Med Biol 1974, 45(0), 3-11.

[33] Apter, F.M., Lencer, W.I., Finkelstein, R.A., Mekalanos, J.J. \& Neutra, M.R. Monoclonal immunoglobulin A antibodies directed against cholera toxin prevent the toxin-induced chloride secretory response and block toxin binding to intestinal epithelial cells in vitro. Infect Immun 1993, 61(12), 5271-5278.

[34] Mazanec, M.B., Kaetzel, C.S., Lamm, M.E., Fletcher, D. \& Nedrud, J.G. Intracellular neutralization of virus by immunoglobulin A antibodies. Proc Natl Acad Sci U S A 1992, 89(15), 6901-6905.

[35] Stephens, D. \& Farley, M. Pathogenic events during infection of the human nasopharynx with Neisseria meningitidis and Haemophilus influenzae. Rev Infect Dis 1991, 13(1), 22-33.

[36] Virji, M., Makepeace, K., Ferguson, D.J., Achtman, M. \& Moxon, E.R. Meningococcal Opa and Opc proteins: their role in colonization and invasion of human epithelial and endothelial cells. Mol Microbiol 1993, 10(3), 499-510.

[37] Yazdankhah, S.P. \& Caugant, D.A. Neisseria meningitidis: an overview of the carriage state. J Med Microbiol 2004, 53(Pt 9), 821-832.

[38] Stern, A. \& Meyer, T.F. Common mechanism controlling phase and antigenic variation in pathogenic neisseriae. Mol Microbiol 1987, 1(1), 5-12.

[39] Cho, Y., Usui, K., Honda, S., Tahara-Hanaoka, S., Shibuya, K. \& Shibuya, A. Molecular characteristics of IgA and IgM Fc binding to the Fcalpha/muR. Biochem Biophys Res Commun 2006, 345(1), 474-478.

[40] Schneider, C., Owen, M.J., Banville, D. \& Williams, J.G. Primary structure of human transferrin receptor deduced from the mRNA sequence. Nature 1984, 311(5987), 675678.

[41] Diven, S.C., Caflisch, C.R., Hammond, D.K., Weigel, P.H., Oka, J.A. \& Goldblum, R.M. IgA induced activation of human mesangial cells: independent of FcalphaR1 (CD 89). Kidney Int 1998, 54(3), 837-847.

[42] Kitamura, T., Garofalo, R.P., Kamijo, A. et al. Human intestinal epithelial cells express a novel receptor for IgA. J Immunol 2000, 164(10), 5029-5034. 
[43] Mantis, N.J., Cheung, M.C., Chintalacharuvu, K.R., Rey, J., Corthesy, B. \& Neutra, M.R. Selective adherence of IgA to murine Peyer's patch M cells: evidence for a novel IgA receptor. J Immunol 2002, 169(4), 1844-1851.

[44] Barratt, J., Greer, M.R., Pawluczyk, I.Z. et al. Identification of a novel Fcalpha receptor expressed by human mesangial cells. Kidney Int 2000, 57(5), 1936-1948.

[45] Sixbey, J.W. \& Yao, Q.Y. Immunoglobulin A-induced shift of Epstein-Barr virus tissue tropism. Science 1992, 255(5051), 1578-1580.

[46] Weiser, J.N., Bae, D., Fasching, C., Scamurra, R.W., Ratner, A.J. \& Janoff, E.N. Antibody-enhanced pneumococcal adherence requires IgA1 protease. Proc Natl Acad Sci U S A 2003, 100(7), 4215-4220.

[47] Vidarsson, G., Overbeeke, N., Stemerding, A.M. et al. Working mechanism of immunoglobulin A1 (IgA1) protease: cleavage of IgA1 antibody to Neisseria meningitidis PorA requires de novo synthesis of IgA1 Protease. Infect Immun 2005, 73(10), 6721-6726.

[48] Findlow, J., Taylor, S., Aase, A. et al. Comparison and correlation of neisseria meningitidis serogroup B immunologic assay results and human antibody responses following three doses of the Norwegian meningococcal outer membrane vesicle vaccine MenBvac. Infect Immun 2006, 74(8), 4557-4565.

[49] Virji, M., Makepeace, K., Peak, I.R., Ferguson, D.J., Jennings, M.P. \& Moxon, E.R. Opc- and pilus-dependent interactions of meningococci with human endothelial cells: molecular mechanisms and modulation by surface polysaccharides. Mol Microbiol 1995, 18(4), 741-754.

[50] Fredriksen, J.H., Rosenqvist, E., Wedege, E. et al. Production, characterization and control of MenB-vaccine "Folkehelsa": an outer membrane vesicle vaccine against group B meningococcal disease. NIPH Ann 1991, 14(2), 67-79; discussion 79-80.

[51] Tobin, R., Staehlin, T. \& Gordon, J. Electrophoretictransfer of protein from polyacrylamide gels to nitrocellulose sheets: procedure and some applications. Proc. Nat. Acad. Sci. U.S.A. 1979, 76, 4350-4354.

[52] Blanch, V.J., Piskurich, J.F. \& Kaetzel, C.S. Cutting edge: coordinate regulation of IFN regulatory factor-1 and the polymeric Ig receptor by proinflammatory cytokines. $J$ Immunol 1999, 162(3), 1232-1235.

[53] Dixon, G.L., Heyderman, R.S., Kotovicz, K. et al. Endothelial adhesion molecule expression and its inhibition by recombinant bactericidal/permeability-increasing protein are influenced by the capsulation and lipooligosaccharide structure of Neisseria meningitidis. Infect Immun 1999, 67(11), 5626-5633.

[54] Kues, W.A., Anger, M., Carnwath, J.W., Paul, D., Motlik, J. \& Niemann, H. Cell cycle synchronization of porcine fetal fibroblasts: effects of serum deprivation and reversible cell cycle inhibitors. Biol Reprod 2000, 62(2), 412-419.

[55] Tsai, C.M., Frasch, C.E. \& Mocca, L.F. Five structural classes of major outer membrane proteins in Neisseria meningitidis. J Bacteriol 1981, 146(1), 69-78.

[56] Kitamura, T., Garofalo, R., Kamijo, A. et al. Human intestinal epithelial cells express a novel receptor for IgA. J Immunol 2000, 15(164), 5029-5034.

[57] Kaetzel, C., Robinson, J., Chintalacharuvu, K., Vaerman, J. \& Lamm, M. The polymeric immunoglobulin receptor (secretory component) mediates transport of immune complexes across epithelial cells: a local defense function for IgA. Proc Natl Acad Sci U S A. 1991, 88(19), 8796-8800. 
Figure 1

a)

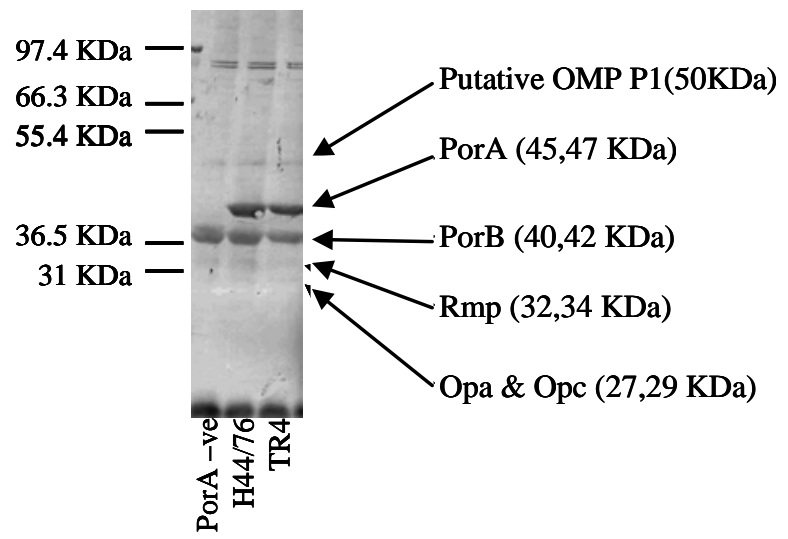

b)

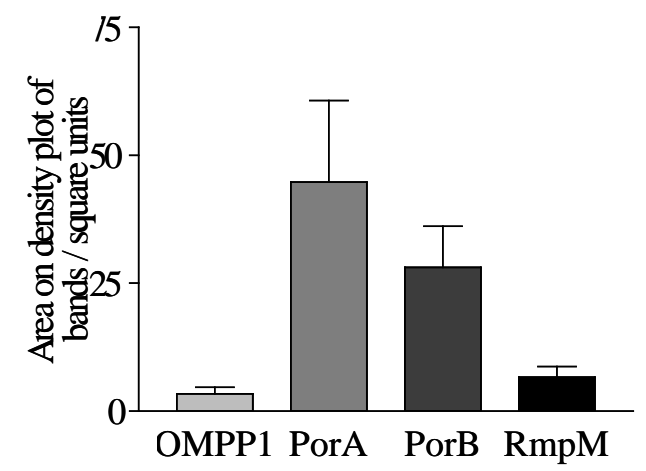


Figure 2

a) Association time course

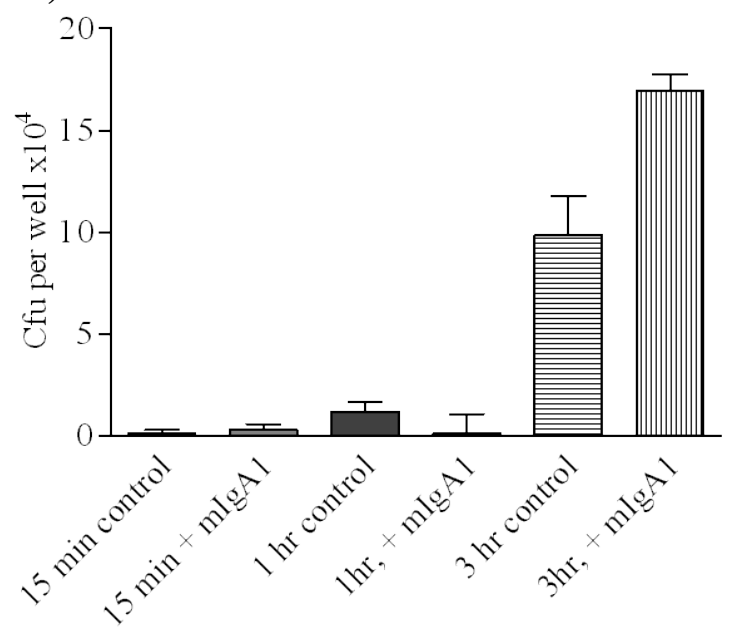

b) Association with Fab fragments

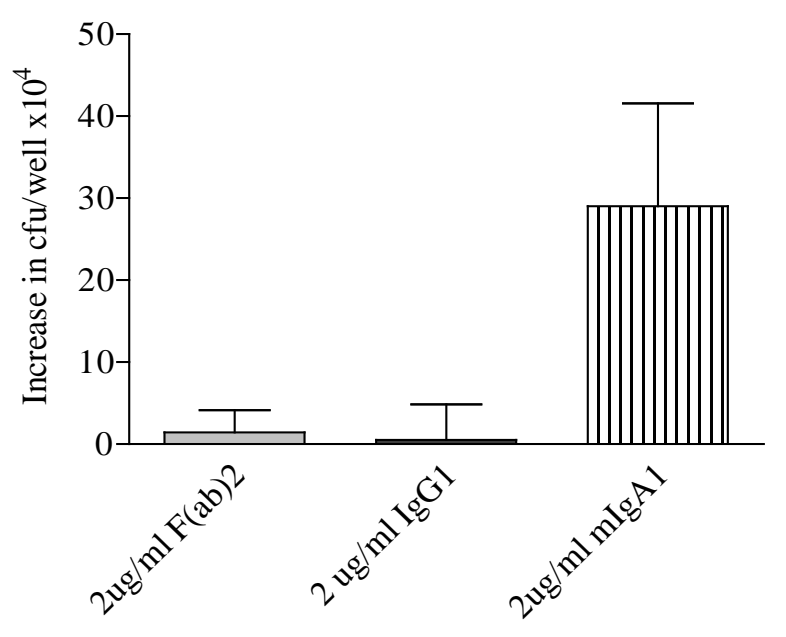


Figure 3

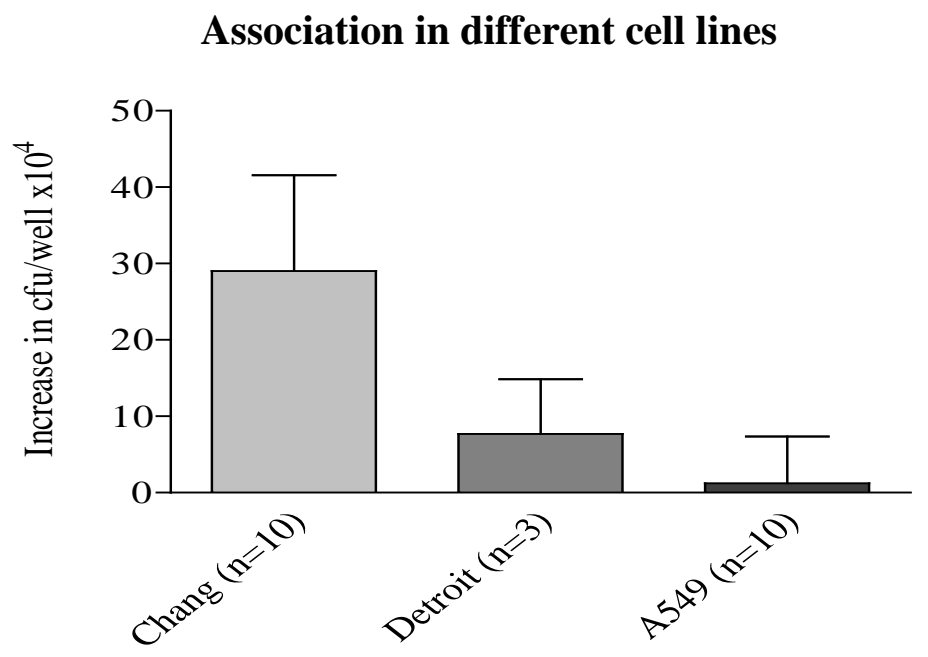



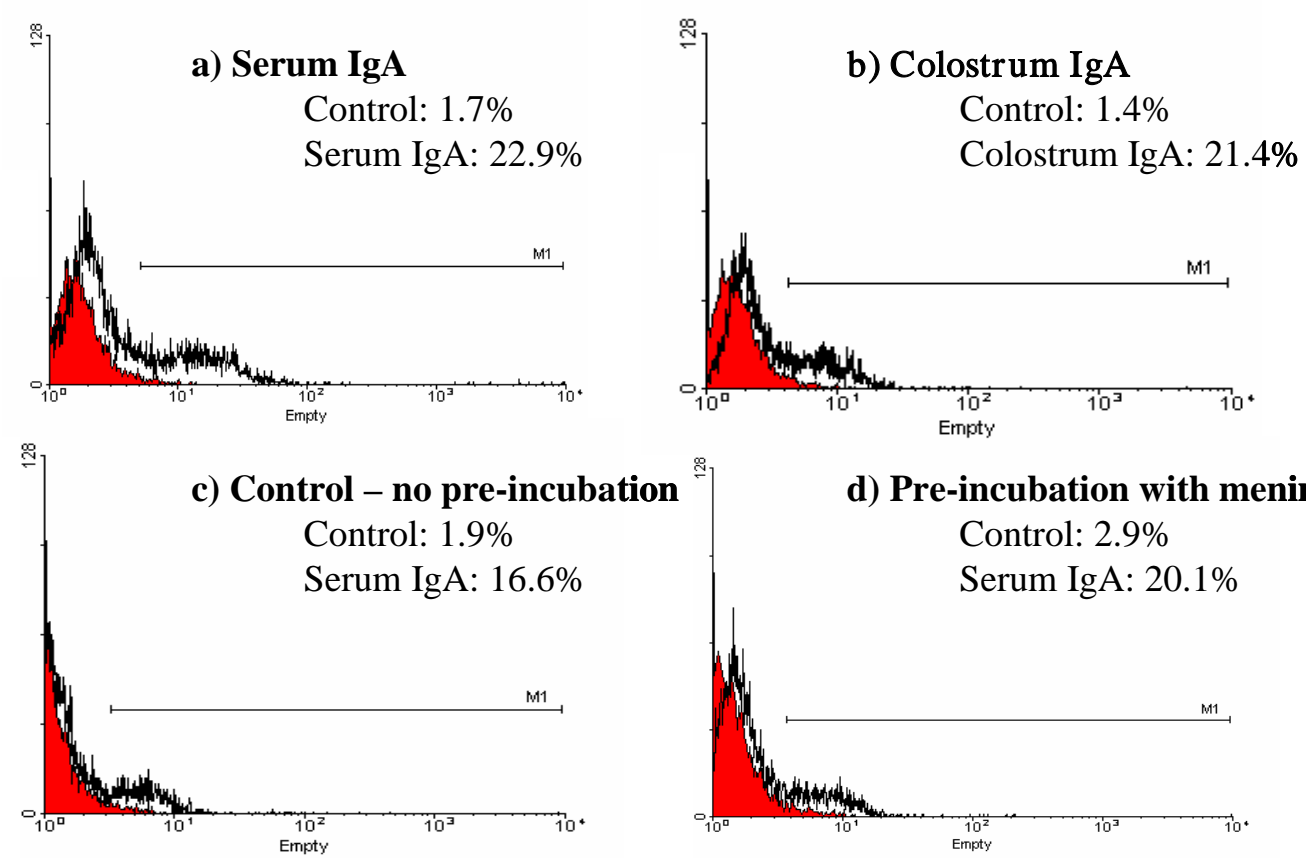

d) Pre-incubation with meningococci Control: $2.9 \%$

Serum IgA: $20.1 \%$
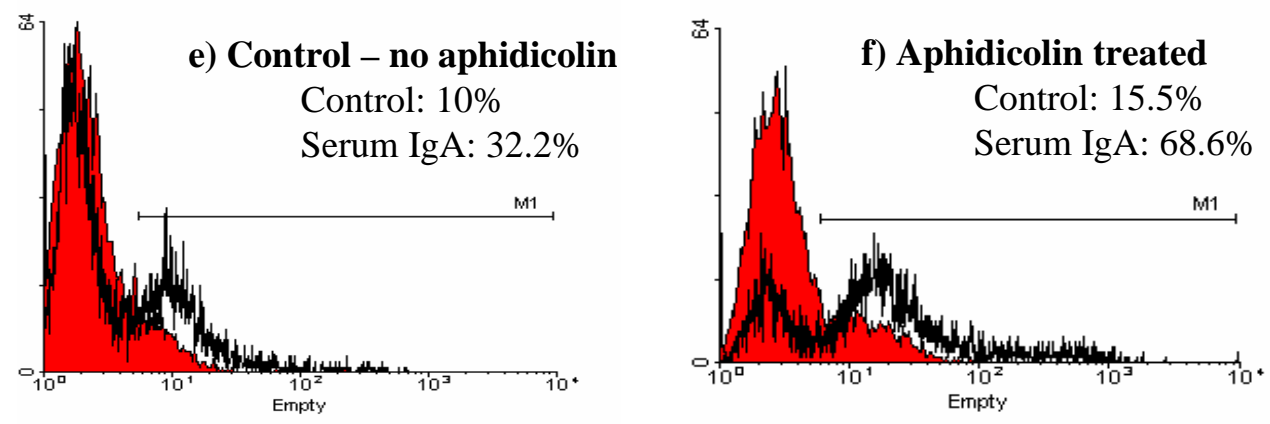
Figure 5

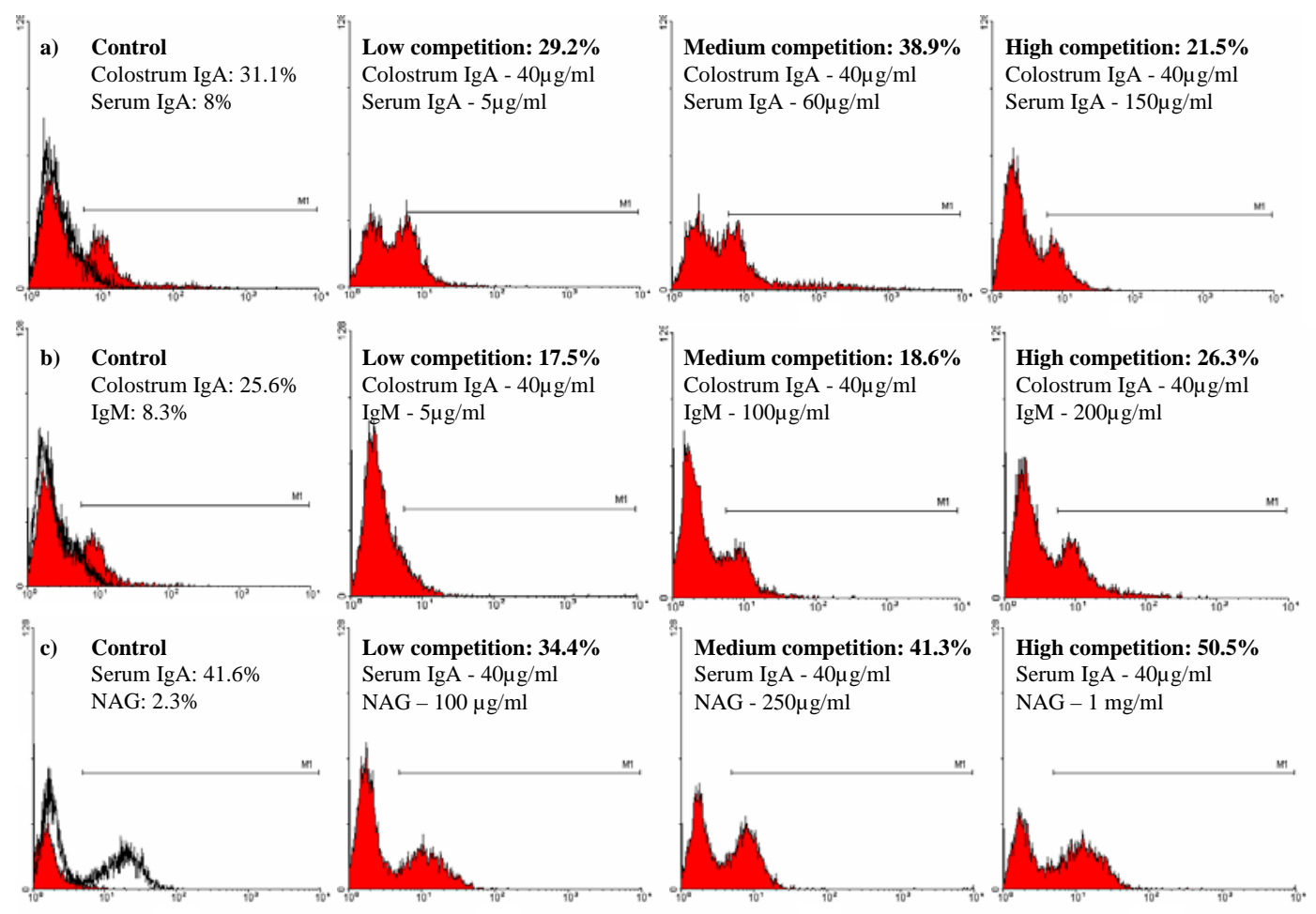


Figure 6

a)

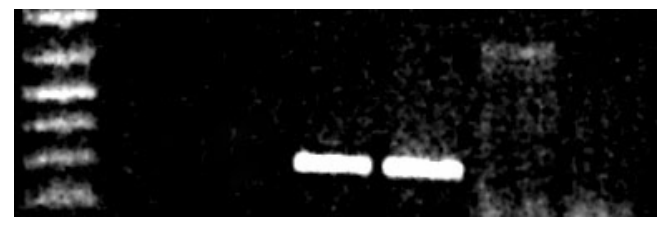

FcaR (797bp)

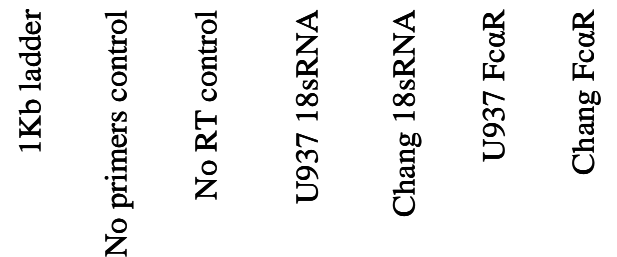

b)

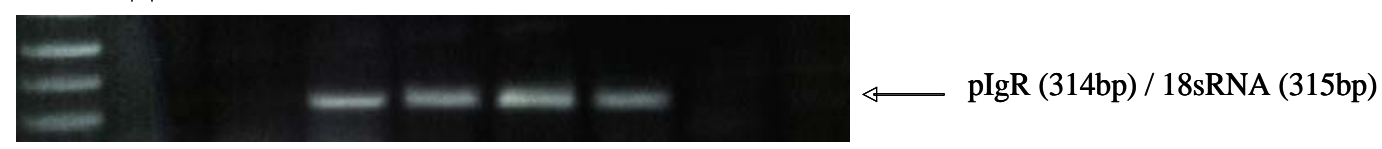

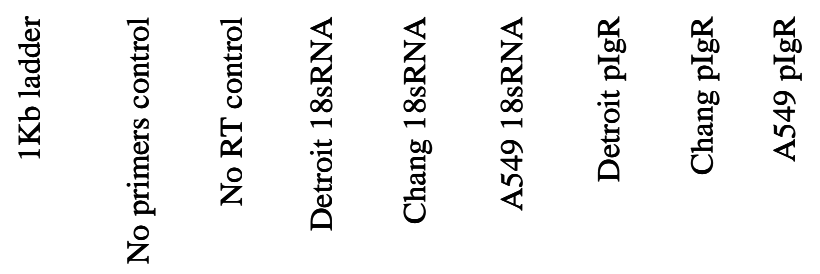




\section{Figure Legends}

Figure 1: Meningococcal antigen specificity of salivary IgA

a) Western blot with saliva from a representative individual. From left to right, the lanes contain the PorA negative OMV, WT H44/76, and WT TR4. OMP indicated are based on molecular weights : Putative OMP P1 (50kDa), PorA (46Kda \pm 1), PorB (41/38 KDa \pm 1), Rmp (33Kda \pm 1) and Opa/Opc (28 KDa \pm 1) [55].

b) Density plot of Western blot bands. Columns represent the mean of 5 individual blots measuring anti-meningococcal salivary antibody specificities, 2 children and 3 adults. SEM is shown by vertical bars.

Figure 2: The effect of antibody on meningococcal association with Chang cells. Meningococcal H44/76 suspensions with or without anti-PorA antibody were incubated with epithelial cell monolayers at $37^{\circ} \mathrm{C}$ for periods shown (a) or three hours (b). Adherent bacteria were enumerated as described in methods by viable count assays.

a) Time course of the association of $\mathrm{H} 44 / 76$ meningococci with Chang cells with and without the presence of mIgA1 $(2 \mu \mathrm{g} / \mathrm{ml})$. Little cell association was observed at earlier time points and no discernable difference between control and test samples could be seen. However at 3 hours significantly more meningococci were associated with Chang cells in the presence of mIgA1 than in control wells $(\mathrm{P}=0.0435)$.Columns represent the means of triplicate determinations with SEM shown by the vertical lines. The graph is a representative example of data from three separate experiments.

b) Effect of IgG1 and the $F(a b)_{2}$ portions of mIgA1 on meningococcal association with Chang cells. In contrast to mIgA1, no increase in meningococcal association was seen with either $\mathrm{F}(\mathrm{ab})_{2}(\mathrm{SEM}=1.425 \pm 2.721)$, or IgG1 (SEM=0.5025 \pm 4.334$)$. For each experiment the 
control values (no antibody) have been subtracted from the results so the bars represent association above background. Means and SEM of at least three experiments are shown.

Figure 3: The effect of mIgA1 on meningococcal association with different cell lines. Meningococcal association with Chang cells when mIgA1 is present $(\mathrm{P}=0.05)$, but this increase is not seen with either Detroit $(\mathrm{P}=0.9314)$ or A549 cells $(\mathrm{P}=0.7535)$. Each column represents the mean of at least three experiments. For each experiment the control values (no antibody) have been subtracted from the results so bars represent association above background. SEM is shown by the vertical bars.

Figure 4: Both serum and secretory IgA bind to Chang cells and binding efficiency depends of the cell cycle. IgA derived from both serum (a) and colostrum (b) binds to a subpopulation of Chang cells. The population of cells bound by serum derived IgA is increased when cells are treated with the cell cycle inhibitor aphidicolin (e\&f) but not by preincubation with meningococci (c\&d). Flow cytometry was carried out on cells which were incubated with biotinylated IgA preparations for 20 minutes on ice, then blocked and incubated for a further 20 minutes on ice with secondary antibody (streptavidin-FITC conjugate). Binding by streptavidin-FITC is shown in red. Each histogram shown is representative of at least three individual experiments. IgG was used as an isotype control and did not bind Chang cells (data not shown).

Figure 5: Characteristics of IgA binding to Chang cells. Neither mIgA (a) nor IgM effect the binding of pIgA to Chang cells, and no inhibition of mIgA binding is seen with N-acetyl- 
D-galactosamine (NAG) (c). Flow cytometry was carried out on cells which had been incubated with biotinylated colostrums (a\&b) or serum (c) derived IgA for 20 minutes on ice, simultaneously with serum derived IgA (a), IgM (b) or NAG (c), then blocked and incubated for a further 20 minutes on ice with FITC conjugated streptavidin (shown in red). Binding of non-biotinylated competing reagents, used as negative controls, is shown by the black line. All histograms shown are representative of three individual experiments.

Figure 6: Agarose gels showing the expression of the FcaR (a) and pIgR(b). Total RNA was extracted from cells using RNAqueous-4PCR (Ambion, Texas, USA) and RT-PCR carried out using the Access RT-PCR system (Promega). Positive control for Fc $\alpha$ R is PMA stimulated U937 cells [56], and for pIgR is Detroit cells with A549 cells as pIgR negative control [57]. 